\title{
Puentes mucosos de la cuerda vocal, en la Unidad de Voz del Hospital Clínico de la Universidad de Chile: diagnóstico y manejo desafiante
}

\section{Mucosal bridges in Voice Unit at the Clinical Hospital Universidad de Chile: challenging diagnosis and management}

\author{
Karen García C. ${ }^{1}$, Daniela Vicencio S. ${ }^{2}$, Pedro Cortez V. ${ }^{3}$, Jorge Zúñiga P. ${ }^{3}$, Christian Olavarría L. ${ }^{3}$
}

\section{Resumen}

Introducción: Los puentes mucosos (PM) corresponden a una banda de mucosa paralela al borde libre de la cuerda vocal, unido por anterior y posterior como un ojal. Su manejo no está estandarizado y la literatura al respecto es escueta, con cirugías con resultados no siempre predecibles. Objetivo: Descripción de las características clínicas de pacientes con PM y las técnicas quirúrgicas para su manejo, en la Unidad de Voz del Servicio de Otorrinolaringología del Hospital Clínico de la Universidad de Chile (HCUCH), discutiendo el desafío para su diagnóstico y manejo. Material y Método: Estudio retrospectivo, descriptivo, incluyendo pacientes con PM, entre los años 2013 y 2019 en HCUCH. La evaluación preoperatoria incluye anamnesis, examen físico, nasofibroscopía y/o telelaringoscopía, junto con estroboscopía. Resultados: Se incluyen 7 casos, con edad promedio de 37,4 años; todas mujeres, con diagnóstico realizado en pabellón, en una sola cuerda vocal. Se asoció a alteración estructural mínima (AEM) en el $100 \%$ de los casos y a patología benigna en $75 \%$. Todos los pacientes consultaron por disfonía. El tratamiento fue elegido de acuerdo a cada paciente, con resección en todos los casos, con variadas técnicas de microcirugía laríngea, con infiltración de corticoides y/o grasa, además de fonoterapia. Conclusión: Los PM, deben sospecharse en disfonía crónica con respuesta no favorable a fonoterapia, en asociación a otras AEM, en particular en mujeres, con diagnóstico en el intraoperatorio con equipos adecuados, incluyendo consentimiento informado para eventual manejo quirúrgico. Es una patología poco frecuente, cuyo manejo no ha sido estandarizado, por lo que nuestra experiencia resulta relevante.

Palabras clave: voz, microcirugía laríngea, alteración estructural mínima, puente mucoso, disfonía.

\begin{abstract}
Introduction: Mucosal bridges $(\mathrm{MB})$ correspond to a mucosa loop parallel to the free edge of the vocal fold, which is joined forward and backward. There is not a standardized procedure for its treatment and the literature in this regard is scarce and surgical management have not resulted in predictable outcomes. Aim: Description of clinical characteristics of patients with MB and the surgical techniques, in the Otorhinolaryngology Service at the Clinical Hospital Universidad de Chile (HCUCH), reflecting upon the challenge for its diagnosis and management. Material and Method: Retrospective and descriptive study, including patients with diagnosis of MB, between 2013 and 2019 in HCUCH. The preoperative evaluation includes anamnesis, physical examination, nasofibroscopy and/or telelaryngoscopy, along with the use of stroboscopy. Results: 7 cases were included, with an average age of 37.4 years; all women, with diagnosis made in the operating room, on a single vocal fold. It was associated with another minimal structural abnormality (MSA) in 100\% of the cases, and with benign pathology in $75 \%$ of them. Dysphonia was the main symptom. The treatment was chosen individually, with resection in all cases, various laryngeal microsurgery tech-
\end{abstract}

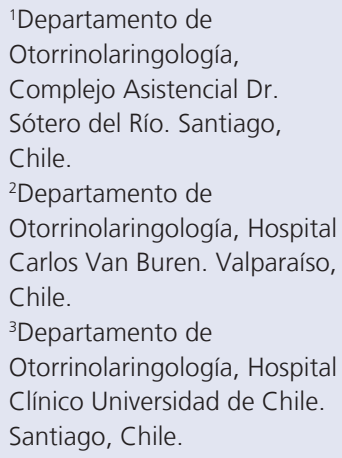

Los autores declaran no tener conflictos de interés.

Recibido el 23 de abril de 2020. Aceptado el 21 de septiembre de 2020 .

Correspondencia:

Christian Olavarría L.

Avenida Dr. Carlos Lorca

Tobar 999,

Departamento de Otorrinolaringología Hospital Clínico Universidad de Chile.

Santiago, Chile.

Email: christian.olavarria@ gmail.com 
niques, infiltration of steroids and/or fat, in addition to speech therapy. Conclusion: $M B$ should be suspected in cases of chronic dysphonia with an unfavorable response to speech therapy, in association with other MSAs, particularly in women, diagnosed intraoperatively with adequate equipment, including informed consent for eventual surgical management. It is a rare pathology, whose management has not been standardized therefore our experience is relevant.

Keywords: voice, laryngeal microsurgery, minimal structural alteration, mucosal bridge, dysphonia.

\section{Introducción}

El término alteraciones estructurales mínimas (AEM) fue utilizado por primera vez por Paulo Pontes en 1994. Éstas corresponden a una serie de variaciones anatómicas mínimas, o pequeñas malformaciones limitadas a las cuerdas vocales, sin otra alteración morfológi$\mathrm{ca}$ asociada ${ }^{1}$. Incluyen diferentes alteraciones, siendo la más frecuente el sulcus vocal y la menos frecuente el puente mucoso (PM), en la que se centra esta publicación ${ }^{2-4}$. Estas corresponden a una banda de mucosa paralela al borde libre de la cuerda vocal, que está unido por anterior y posterior como un ojal. Se compone de un eje central de tejido conectivo denso vascularizado, cubierto por epitelio escamoso no queratinizado, con membrana basal engrosada ${ }^{4-6}$. De acuerdo con la mayoría de los autores, el PM corresponde a una lesión congénita ${ }^{1,4}$, sin embargo, también se ha postulado que se podría desarrollar como complicación de microcirugía laríngea ${ }^{6,7}$. Respecto a los PM la literatura es limitada, con reportes anecdóticos inmersos en series de casos de otras patologías y con escasas series de casos dedicadas en forma exclusiva a este tópico. $\mathrm{Su}$ manejo no está estandarizado y el resultado quirúrgico no siempre es predecible, motivo por el cual consideramos relevante el trabajo que se presenta a continuación.

\section{Objetivo}

Descripción de las características clínicas de pacientes con PM y las técnicas quirúrgicas para su manejo en la Unidad de Voz del Servicio de Otorrinolaringología (ORL) del Hospital Clínico de la Universidad de Chile $(\mathrm{HCUCH})$, discutiendo el desafío para su diagnóstico y manejo.

\section{Material y Método}

Estudio retrospectivo, descriptivo, en base a revisión de ficha clínica, incluyendo pacientes evaluados en Unidad de Voz del HCUCH, con diagnóstico de AEM y de éstos, aquellos con PM. El período de estudio comprende entre los años 2013 y 2019.

La evaluación inicial y de seguimiento en Unidad de Voz incluye anamnesis completa, examen físico, nasofibroscopía y/o telelaringoscopía con luz halógena y estroboscopía, registro visual y de voz. El diagnóstico intraoperatorio incluye el uso de laringoscopía de suspensión con protección dental, junto con endoscopio rígido de $0^{\circ}$ de $4 \mathrm{~mm}, 22 \mathrm{~cm}$, marca Olympus ${ }^{\circledR}$ y/o microscopio, con lente 400, marca Zeiss ${ }^{\circledR}$, con observación directa y palpación. Para la intubación se emplean tubos orotraqueales de microcirugía laríngea. La cirugía es realizada por un miembro de equipo de ORL de la Unidad de Voz del $\mathrm{HCUCH}$.

Se incluyeron todos los pacientes evaluados en la Unidad de Voz del HCUCH, con diagnóstico de AEM, sometidos a cirugía en nuestra institución, en la que se pesquisara PM, con registro de este hallazgo en el protocolo quirúrgico. De aquellos pacientes con PM, no se excluyó ninguno. El análisis de datos se realizó con Microsoft Excel ${ }^{\circledR}$. Este trabajo cuenta con la aprobación del comité de ética del HCUCH.

\section{Resultados}

En el período de estudio, 101 pacientes fueron diagnosticados con AEM en la Unidad de Voz del HCUCH. De éstos, la mayoría tiene más de una AEM. Con distribución por sexo de 61 mujeres y 40 hombres, la edad promedio 
al diagnóstico fue de 35,7 años, con 39,8 años para las mujeres y 30,6 años para los hombres.

La selección de pacientes para realización de microcirugía laríngea se basa en un análisis completo y multidisciplinario, en base a la repercusión en la función laríngea, calidad de vida y ausencia de respuesta a fonoterapia. Del total de pacientes, 50 fueron sometidos a cirugía, instancia en la cual, en 7 pacientes se agregó el diagnóstico de PM, en ninguno de los cuales había sido realizado diagnóstico previo a la laringoscopía directa en pabellón (Figura 1).

Estos pacientes fueron incluidos en el estudio y, sobre estos, se presentan los resultados a continuación. El total de pacientes con PM fueron de sexo femenino, con edad promedio al diagnóstico de 37,4 años, con rango de 18 a 52 años. Dentro de las comorbilidades médicas destaca: tabaquismo en dos pacientes y reflujo faringolaríngeo en cinco de ellos. Ninguno de los pacientes tenía antecedentes de intubación orotraqueal o microcirugía laríngea previa. Las ocupaciones de las pacientes corresponden a: estudiante de música (2), cantante (1), dueña de casa (2), profesora de básica (1) e ingeniera en un banco (1).

Los síntomas referidos por los pacientes en la primera consulta son disfonía en el 100\%, quiebres vocales y fatiga vocal en el $28,6 \%$ de los casos para ambos síntomas. La lateralidad fue 4 derecha, 3 izquierda, 0 bilateral. En 1 caso el PM fue doble en una misma cuerda vocal. En 100\% se asoció a otra AEM: principalmente sulcus $(100 \%)$ y vasculodisgenesia tanto ipsi como contralateral $(71,4 \%)$. En dos casos se asoció a patología benigna de cuerda vocal; siendo en ambos, pólipo cordal y ninguno a patología maligna.
El tratamiento de la AEM diagnosticada en forma ambulatoria, fue elegido de acuerdo a cada paciente en base a la decisión en Unidad de Voz y considerando el uso vocal, deseos y expectativas de los pacientes. Todas las pacientes recibieron fonoterapia pre y posoperatoria. La microcirugía laríngea se realizó con endoscopio de $0^{\circ}$ en forma exclusiva en 4 casos y, combinado con microscopio, en 3 casos.

En el total de los casos en la sala de operaciones, el PM y la AEM asociada fue manejada quirúrgicamente. En un inicio, luego de la adecuada exposición con laringoscopía de suspensión, se palparon meticulosamente ambas cuerdas vocales y se realizó un diagnóstico certero. En 3 casos se realizó resección simple del puente mucoso, con microtijera, a nivel de la unión superior e inferior del PM con el resto de la cuerda vocal (CV). En los 4 restantes se optó por disección de mucosa interna. Se identifica el PM en su totalidad, se decola la mucosa, tanto del PM como de la cuerda vocal. El PM no se secciona, y se posicionan ambas mucosas, afrontándolas entre sí, requiriendo sutura con vycril 7.0 en 2 de ellos. Además, posterior a la remoción del PM, 2 requirieron infiltración con grasa, y en los 5 casos restantes se infiltró con corticoide de depósito (Depo-Medrol) en el espacio de Reinke. En cuanto al posoperatorio: todas las pacientes fueron dadas de alta el mismo día de la cirugía. Se indicó al alta reposo vocal absoluto por 7 días, uso de inhibidores de la bomba de protones y papaína. El primer control se realizó en los primeros 7 a 10 días posoperatorios. No se presentó ninguna complicación intraoperatoria, derivada de la LD o del procedimiento quirúrgico, ni posoperatorias.

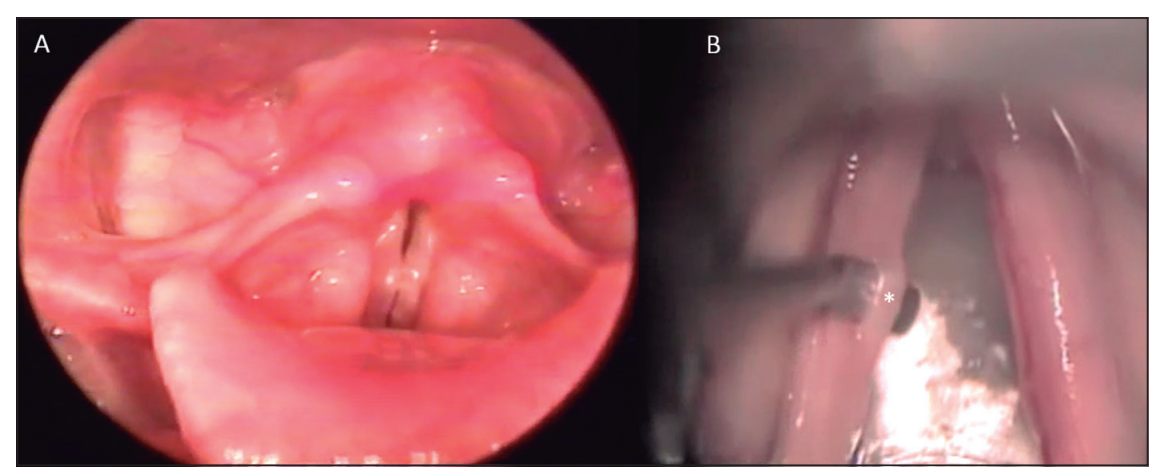

Figura 1. Comparación de visualización con telelaringoscopía (A) y con laringoscopía directa en pabellón (B), donde se evidencia con palpación puente mucoso $\left(^{*}\right)$ en cuerda vocal derecha, en el mismo sujeto. 
El valor promedio de la encuesta Voice Handicap Index previo y posterior a la microcirugía laríngea del PM fue de 31 y 22 respectivamente. El seguimiento corresponde a 12,25 meses en promedio. Se debe especificar que una paciente requirió una reintervención 5 años después para infiltración de grasa.

\section{Discusión}

En cuanto a la epidemiología de nuestra serie, el total de la muestra de pacientes con PM corresponde al sexo femenino. Si bien, el número es pequeño, nos parece relevante mencionar que pareciera haber una tendencia a la afectación en mujeres. Sin embargo, en las otras series reportadas de PM, encontramos resultados que discrepan entre sí. También resaltamos que en la serie solo hay pacientes adultos, lo cual se condice con otras casuísticas, encontrando solo tres casos en paciente pediátrico ${ }^{5,6}$.

En la anamnesis resulta relevante preguntar dirigidamente por la ocupación del paciente, más aún si consulta por disfonía, para estimar el uso vocal y cómo la disfonía podría afectar su calidad de vida. Además, en pacientes con uso profesional de la voz, las AEM representan el $24 \%$ a $40 \%$ de las lesiones observadas en endoscopía ${ }^{8}$.

Respecto a la sintomatología clínica, todas las pacientes refieren disfonía de larga data. Por otro lado, todas las pacientes tienen, al menos, una consulta previa con ORL. Esta característica de tiempo prolongado previo al diagnóstico en Unidad de Voz también se repite en nuestra casuística de parálisis cordal. Por lo que la falta de diagnóstico preciso prolonga el tiempo en que los pacientes no son resueltos correctamente 9 . Sumado a esto, la severidad de la disfonía dependerá del grosor del PM, así como de otras AEM o patología benigna asociada. La disfonía de larga data apoya su origen congénito, así como la asociación a otras AEM.

En un trabajo retrospectivo anterior del HCUCH, entre los años 1997 y 2001, 48 pacientes fueron diagnosticados con AEM, y no se encontró ninguno con $\mathrm{PM}^{10}$. No encontramos otras referencias en la literatura nacional respecto a la epidemiología de las AEM y de cada una de ellas. En la literatura internacional, el 1,5\% a $8 \%$ del total de pacientes sometidos a microcirugía laríngea por patología benigna, corresponde a PM, y el porcentaje obtenido en nuestra casuística, de 6,9\%, es similar, calculado en base a pacientes con AEM sometidos a microcirugía laríngea ${ }^{2,11,12}$.

Resaltamos que dos pacientes también tenían como diagnóstico pólipo de cuerda vocal. La asociación entre pólipo de cuerda vocal y AEM ya había sido descrita en la literatura ${ }^{1,12,13}$. Sakae y cols., realizaron una revisión retrospectiva de 68 pacientes sometidos a cirugía por pólipo de cuerda vocal. El 23,5\% tenía una AEM asociada, siendo en el 69\% de los casos, contralateral al pólipo. Solo el 6,2\% correspondía a $\mathrm{PM}^{12}$. También se ha descrito su asociación a otras AEM, en su mayoría a sulcus vocalis, al igual que nuestra serie $e^{1,4-6,14}$.

Diversos estudios concluyen que existe una inconsistencia entre el diagnóstico pre e intraoperatorio para lesiones cordales benignas, siendo consistente solo en el $34 \%$ a $64 \%$ de los casos. En el 65\% a 67\% de los pacientes, el diagnóstico es modificado en pabellón o se agrega una lesión que no fue pesquisada en la evaluación ambulatoria inicial. En un 31\% se agrega diagnóstico en ambas cuerdas vocales. Un gran porcentaje de las lesiones adicionales (23\%-93\%) involucran AEM, en particular el PM. En nuestra serie, todos los casos de PM fueron pesquisados en pabellón, lo cual también está descrito en trabajos internacionales ${ }^{2,5,6,11,12,15,16}$.

Postulamos que un factor relevante para obviar el diagnóstico de PM en forma ambulatoria, es que en la cirugía, las cuerdas vocales se tensan por el uso de laringoscopía de suspensión, lo cual modifica la visión de éstas y permite observarlas completamente, $y$ palparlas, lo cual, evidentemente, no es posible de realizar en forma ambulatoria, lo cual también ha sido planteado por otros autores ${ }^{2,4,6,11}$.

Otro motivo que postulamos como dificultad diagnóstica es que, en el análisis ambulatorio, si bien la estroboscopía se menciona en forma general como fundamental, para el caso específico de PM la característica estroboscópica principal es la disminución de la vibración con severidad variable, lo cual se puede atribuir a otras patologías visibles en consulta ambulatoria. Se hace más evidente al solicitar inspiración profunda al paciente u olfateo. El 
segmento de mucosa correspondiente al PM no vibra junto con el resto de la $\mathrm{CV}$. Incluso al revisar en forma retrospectiva las estroboscopías de pacientes con PM, los observadores no pudieron identificarlos. Por otro lado, el aspecto endoscópico del PM es muy similar al del sulcus vocal, lo cual dificulta también su diagnóstico ${ }^{5,6,11}$.

Pese a la evaluación preoperatoria con luz halogenada y estroboscópica, endoscopía flexible y rígida, con más de un observador, el diagnóstico de PM aún sigue siendo complejo. Por lo tanto, un examen minucioso durante la microcirugía laríngea, de ambas cuerdas vocales, con inspección y palpación no traumática es fundamental para modificar, agregar o corroborar el diagnóstico preoperatorio ${ }^{2,6,17}$. En la inspección, el PM puede ser palpado con gancho romo y separado con micro fórceps laríngeo, y traccionado hacia la línea media, para diferenciarlo del resto de la cuerda vocal.

Esto refleja la importancia de ser conscientes de la posibilidad de modificación del diagnóstico en la sala de operaciones, lo cual debe ser apropiadamente informado al paciente $y$, en consecuencia, la eventual modificación de la planificación quirúrgica, tanto en diagnóstico de la patología específico, así como de la lateralidad de ésta. Creemos que esto debe ser conversado y comprendido tanto por el equipo de ORL, así como por el paciente, y el consentimiento informado debe incluir esta posibilidad. Los puntos clave sugeridos para el consentimiento informado de pacientes sometidos a microcirugía laríngea se resumen en la Tabla $1^{18}$.

Al revisar los videos y protocolos quirúrgicos, realizamos una caracterización de los PM. Suelen corresponder a lesiones únicas, con solo un caso con afectación múltiple, unilaterales ( 0 casos bilaterales), ubicadas en la cara superior de la cuerda vocal, paralelas al borde libre de la CV, asociada generalmente a otra AEM; sulcus y vasculodisgenesia en su mayoría, y a lesión benigna como pólipo, con largo y grosor variable, ubicado en general en tercio medio de la CV (Tabla 2 y Figura 2). En la literatura solo encontramos ocho casos que reporta bilateralidad y con menor frecuencia aún, multiplicidad de $\mathrm{PM}$ en el mismo paciente $\mathrm{e}^{6,11,17,19,20}$.

Respecto al tratamiento, existe escasa experiencia publicada. Podría manejarse en

\begin{tabular}{l}
$\begin{array}{l}\text { Tabla 1. Características de los puentes } \\
\text { mucosos analizados en esta serie }\end{array}$ \\
\hline Única \\
Unilateral \\
Cara superior de la cuerda vocal \\
Paralela al borde libre \\
Asociada a otra alteración estructural mínima \\
Grosor variable \\
Largo variable \\
En tercio medio de la cuerda vocal
\end{tabular}

Tabla 2. Elementos clave a considerar en el consentimiento informado e información para el paciente previo a microcirugía laríngea. Adaptado desde Zeitels y cols. ${ }^{18}$

No se es posible garantizar mejoría de la disfonía

Existe la posibilidad de empeoramiento de la voz

Modificación del diagnóstico

Hallazgos en el intraoperatorio pueden modificar la conducta quirúrgica

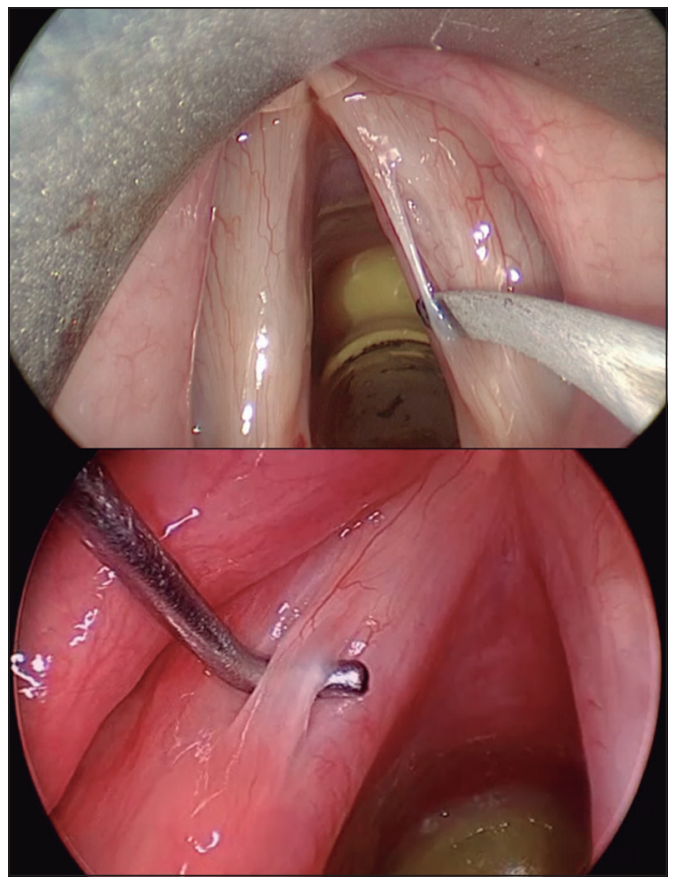

Figura 2. Imágenes de puentes mucosos en el intraoperatorio con laringoscopía de suspensión, previo a su respectivo manejo quirúrgico, identificados por palpación. 
forma conservadora con fonoterapia ${ }^{5}$. En la serie presentada, todas las pacientes recibieron esta terapia en primera instancia, y dado el fracaso a ésta, se decidió progresar a la opción de cirugía. Respecto a la resolución quirúrgica, en diversos libros que abordan técnicas quirúrgicas, no se refieren a esta o, si lo hacen, es en forma escueta.

Debido a la falta de información disponible, en base a nuestra experiencia, sugerimos que aquellas lesiones cortas y delgadas, sean removidas con sección simple de ambos extremos para evitar fenómeno de golpe, mientras que en lesiones largas y/o muy anchas, eventualmente sería útil disección de mucosa interna para adherirla a la cuerda vocal, con algún elemento que permita mantenerla en esta posición, pero con riesgo de que, al adherirse, se elimine el defecto de masa, pero produzca rigidez. Puede usarse pegamento de fibrina para estos fines ${ }^{5,6}$. En nuestro centro se prefirió el uso de sutura, técnica recientemente descrita como sandwich mucosal flap surgery ${ }^{6,21}$. También se describe el uso de láser en reemplazo de técnica fría, para reavivar las superficies en contacto, tanto del PM como de la cuerda vocal, con el cual no contamos en nuestro centro, por lo que no tenemos pacientes operados con esta técnica ${ }^{6,22}$.

La remoción del PM puede causar atrofia de la cuerda vocal e incompetencia glótica, por lo que puede utilizarse en forma concomitante diversos injertos para corregirlo ${ }^{5}$. En aquellos casos que fue necesario, se prefirió el uso de grasa. Este trabajo cuenta con la limitación de corresponder a una revisión de carácter retrospectivo, con ausencia de grupo control, por lo que no es posible establecer comparaciones en cuanto a métodos diagnósticos ni técnicas quirúrgicas. Además, resulta complejo realizar un análisis por separado de la técnica empleada, debido a, como discutimos, la alta frecuencia de concomitancia con otras AEM, así como patología benigna. Por otro lado, el número de pacientes incorporados es pequeño. Este trabajo corresponde hasta la fecha, a nuestro entendimiento, a la única casuística reportada de PM en la literatura hispana. En opinión de los autores, y basado en la experiencia debido a falta de información disponible, proponemos un algoritmo de enfrentamiento y manejo, que podría ser mejorado con la contribución de otras series a nivel nacional (Figura 3).

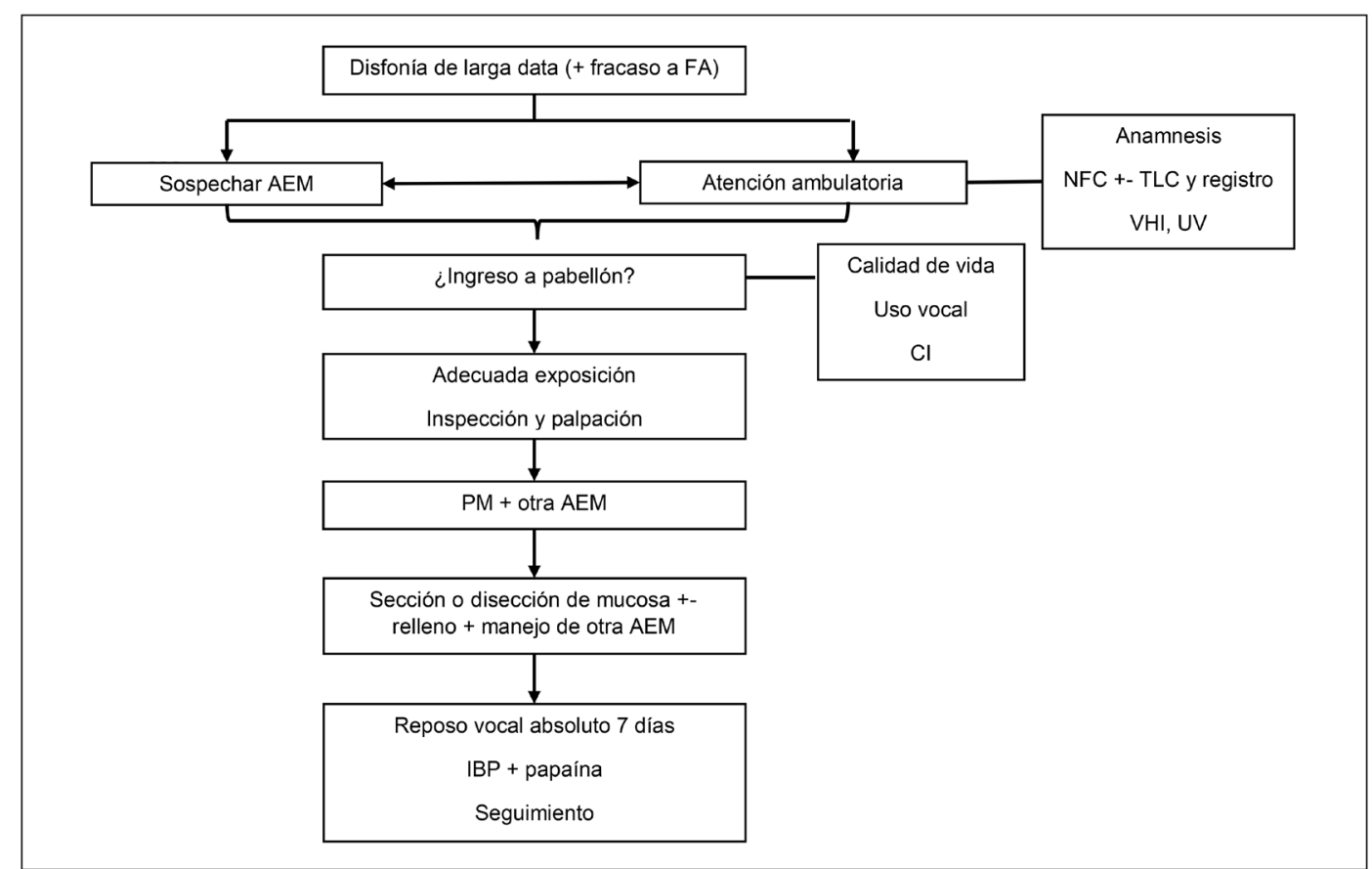

Figura 3. Algoritmo propuesto de enfrentamiento a pacientes con sospecha de alteración estructural mínima y puente mucoso. FA: fonoterapia; AEM: alteración estructural mínima; NFC: nasofibroscopía; TLC: telelaringoscopía; VHI: Voice Handicap Index; UV: unidad de Voz; Cl: consentimiento informado; IBP: inhibidores de la bomba de protones. 


\section{Conclusión}

Los PM forman parte de las AEM, y son subdiagnosticados en la atención ambulatoria de rutina de los pacientes con disfonía. Es una patología poco frecuente, cuyo manejo no ha sido estandarizado, por lo que nuestra experiencia resulta relevante. La evaluación de estos pacientes en Unidad de Voz permite tomar decisiones de mejor manera.

\section{Bibliografía}

1. Pontes P, Behlau M, Goncalves M. Alteraçöes Estruturais Mínimas Da Laringe AEM: Consideraçöes Básicas. Acta Who. 1994;13(1):2-6.

2. Akbulut $\mathrm{S}$, Altintas $\mathrm{H}$, Oguz $\mathrm{H}$.

Videolaryngostroboscopy versus microlaryngoscopy for the diagnosis of benign vocal cord lesions: a prospective clinical study. Eur Arch Otorhinolaryngol. 2015;272(1):131-136. doi: 10.1007/s00405-014-31815.

3. Moraes BT, De Biase NG. Prevalence of Minor Larynx Structural Alterations: Influence on the Concept of a Normal Vocal Fold. Otolaryngol Head Neck Surg. 2019;160(2):295-301. doi: 10.1177/0194599818804768.

4. Bouchayer M, Cornut G, Witzig E, Loire R, Roch JB, Bastian RW. Epidermoid cysts, sulci, and mucosal bridges of the true vocal cord: a report of 157 cases. Laryngoscope. 1985;95(9 Pt 1):1087-1094.

5. Martins RH, Tavares EL, Fabro AT, Martins MG, Dias NH. Mucosal bridge of the vocal fold: difficulties in the diagnosis and treatment. $J$ Voice. 2012;26(1):127-131. doi: 10.1016/j.jvoice.2010.09.004.

6. Nerurkar NK, Sapre A, Gosavi R. Mucosal bridges (MB): a 9-year retrospective study of their incidence with a third variant proposed. Eur Arch Otorhinolaryngol. 2019;276(1):159-165. doi: 10.1007/ s00405-018-5218-7.

7. Kocak I, Aslan G, Dogan M, Comunoglu N. Vocal fold bridge: a complication of a sulcus cyst surgery. J Voice. 2010;24(2):240-241. doi: 10.1016/j. jvoice.2008.07.002.

8. Fortes FS, Imamura R, Tsuji DH, Sennes LU. Profile of voice professionals seen in a tertiary health center. Braz J Otorhinolaryngol. 2007;73(1):27-31. doi: 10.1016/s1808-8694(15)31118-6.

9. Aravena C, Olavarría C, Zúñiga J, Sauvalle M, Cantillano P. Tiroplastía de medialización con GoreTex ${ }^{\circledR}$ : Experiencia de 8 años en Hospital Clínico de la Universidad de Chile. Rev Otorrinolaringol Cir Cabeza Cuello. 2017; 77:9-15. doi: 10.4067/S0718-
48162017000100002.

10. Olavarría C, Brunetto B, Nazar R, Miranda M, Painepán JC. Alteraciones estructurales mínimas: Su rol etiopatogénico en los trastornos vocales. Rev Otorrinolaringol Cir Cabeza Cuello. 2004;64(1):7-12.

11. Dailey SH, Spanou K, Zeitels SM. The evaluation of benign glottic lesions: rigid telescopic stroboscopy versus suspension microlaryngoscopy. J Voice. 2007;21(1):112-118. doi: 10.1016/j.jvoice.2005.09.006.

12. Sakae FA, Sasaki F, Sennes LU, Tsuji DH, Imamura R. Vocal Fold Polyps and Cover Minimum Structural Alterations: Associated Injuries? Rev Bras Otorrinolaringol. 2004;70(6):739-741.

13. Zernotti M, Jackson-Menaldi C, Rubin AD. Vocal fold mucosal bridge without sulcus vocalis in a smoker. Ear Nose Throat J. 2009;88(6):950.

14. Villagomez VJ, Rosen CA. Vocal fold cyst and contralateral occult sulcus mucosal bridge. Ear Nose Throat J. 2000;79(12):910-911.

15. Poels PJ, de Jong FI, Schutte HK. Consistency of the preoperative and intraoperative diagnosis of benign vocal fold lesions. J Voice. 2003;17(3):425-433. doi: 10.1067/s0892-1997(03)00010-9.

16. Neto JAM, Pinna BR, Neto JC, de Sá Pedroso JE. Comparison between telelaryngoscopy and suspension laryngoscopy in the diagnosis of benign vocal fold lesions. Braz J Otorhinolaryngol. 2008;74(6):869-875. doi: 10.1016/S18088694(15)30147-6.

17. Bolzoni Villaret A, Piazza C, Rossini M, Peretti G. Triple mucosal bridge of the glottis. Otolaryngol Head Neck Surg. 2007;137(4):678-679. doi: 10.1016/j. otohns.2007.03.029.

18. Zeitels SM. The Art and Craft of Phonomicrosurgery in Grammy Award-Winning Elite Performers. Ann Otol Rhinol Laryngol. 2019;128(3_suppl):7S-24S. doi: 10.1177/0003489418810697.

19. Tan M, Pitman MJ. A case of bilateral vocal fold mucosal bridges, bilateral trans-vocal fold type III sulci vocales, and an intracordal polyp. J Voice. 2011;25(4):484-486. doi: 10.1016/j.jvoice.2010.01.005.

20. Gull J, Divi V, Ghaderi M, Sataloff RT. Bilateral occult mucosal bridges of the true vocal folds. J Voice. 2009;23(6):733-734. doi: 10.1016/j.jvoice.2008.04.003.

21. Kim H. New surgical technique for mucosal bridge of vocal ford: sandwich mucosal flap surgery. Disponible en: https://es.yesonvc.com/bbs/board.php?bo_ table=work\&wr_id=10. Consultado el 22 de abril de 2020.

22. Chang JW, Park AY, Byeon HK, Choi HS. Use of pulsed dye laser treatments in patients with vocal fold mucosal bridges with sulcus vocalis - our experience of five cases. Clin Otolaryngol. 2017;42(3):715-719. doi: 10.1111/coa.12618. 